\title{
Follicular thyroid tumours: a study of laminin and type IV collagen in basement membrane and endothelium
}

\author{
CH KENDALL, PR SANDERSON, JANET COPE, IC TALBOT \\ From the Department of Pathology, Clinical Sciences Building, Leicester Royal Infirmary, Leicester
}

SUMMARY Immunocytochemical stains for laminin and type IV collagen can be used as markers for basement membrane and vascular endothelium. Thirty four follicular thyroid lesions were examined using these techniques to investigate two aspects: firstly, the relation between the extent of invasion and the integrity of basement membrane; secondly, whether the techniques could enhance the detection of tumour vascular invasion. The results showed that although basement membrane was lost in widely invasive tumours, preservation was seen in most but not all encapsulated tumours. The potential for improved recognition of vascular invasion was also found.

Well differentiated follicular thyroid tumours continue to pose diagnostic problems. The distinction between adenoma and follicular carcinoma can rest on subtle histological changes. For the patient the designation of benign or malignant tumour can have major implications. Treatment may range from lobectomy and reassurance to total thyroidectomy, thyroxine replacement, and the administration of radioactive iodine. Such difficulties have prompted investigation of many other possible discriminating features.

Recently, attention has been focused on the role of basement membrane in the development of invasion. Loss of basement membrane was shown in invasive tumours in many sites. ${ }^{2}$ In the thyroid recent immunofluorescence investigations showed that loss of basement membrane may be found in follicular thyroid tumours. ${ }^{34}$ To investigate the diagnostic potential of this in more detail we examined a larger series of these tumours, using antisera to laminin and type IV collagen, both of which are components of the basement membrane. ${ }^{56}$ The use of these techniques to enhance the detection of tumour vascular invasion was also shown recently.? As vascular invasion by follicular thyroid tumours is considered to be of particular diagnostic importance, and present additional methods for enhancing the detection of vascular invasion are unhelpful, this aspect was also examined.

Accepted for publication 1 July 1985

\section{Material and methods}

Thirty four follicular thyroid tumours were obtaine from the files of the Leicester Royal Infirmary and the Leicester General.Hospital. After examination of sections stained with haematoxylin and eosin these were subdivided by degree of invasion. Twenty one cases showed no evidence of invasion, four showed early invasion of capsule or capsular vessels, and nine widespread invasion. Examples of normal and hyperplastic thyroid were also included.

IMMUNOSTAINING

The tissues were routinely fixed in $10 \%$ formol saline for up to 48 hours and processed in paraffin. After dewaxing $5 \mu \mathrm{m}$ sections were treated for two hours with $0.4 \%$ pepsin in $0.01 \mathrm{~N}$ hydrochloric acid $(\mathrm{pH} 2.0)$ at $37^{\circ} \mathrm{C}$. The sections were then blocked for endogenous peroxidase activity using hydrogen peroxide in methanol. Immunostaining was performed with the peroxidase-antiperoxidase technique using laminin antiserum (1:50 dilution) followed by counterstaining with haematoxylin. The method was derived from that of Ekblom et al. ${ }^{8}$ Rabbit antiserum to EHS tumour laminin was prepared as described by Forster et al. ${ }^{9}$ Eleven cases were also immunostained with antiserum to type IV collagen using the same technique.

FROZEN SECTION MATERIAL

Fresh frozen material was available from five $\frac{\varrho}{\Phi}$ 
Relation of architecture and degree of invasion of follicular tumours to preservation or loss of components of basement membrane

\begin{tabular}{|c|c|c|c|c|c|c|c|c|c|}
\hline \multirow[t]{3}{*}{ Stage of malignancy } & \multicolumn{3}{|c|}{ Follicular areas } & \multicolumn{3}{|c|}{ Trabecular areas } & \multicolumn{3}{|c|}{ Solid areas } \\
\hline & \multirow[t]{2}{*}{ Present } & \multicolumn{2}{|c|}{ Basement membrane } & \multirow[t]{2}{*}{ Present } & \multicolumn{2}{|c|}{ Basement membrane } & \multirow[t]{2}{*}{ Present } & \multicolumn{2}{|c|}{ Basement membrane } \\
\hline & & Preserved & $\begin{array}{l}\text { Partial or } \\
\text { complete loss }\end{array}$ & & Preserved & $\begin{array}{l}\text { Partial or } \\
\text { complete loss }\end{array}$ & & Preserved & $\begin{array}{l}\text { Partial or } \\
\text { complete loss }\end{array}$ \\
\hline \multirow{4}{*}{$\begin{array}{l}\text { Non-invasive lesions } \\
(n=21) \\
\text { Minimally invasive } \\
\text { tumours }(n=4) \\
\text { Widely invasive } \\
\text { tumours }(n=9)\end{array}$} & 19 & 19 & 0 & 2 & 2 & 0 & & & \\
\hline & 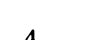 & 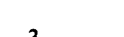 & 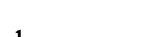 & 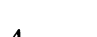 & & & & & \\
\hline & 4 & & 1 & 4 & 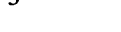 & & & & \\
\hline & 6 & 3 & 3 & 8 & 0 & 8 & 1 & 0 & 1 \\
\hline
\end{tabular}

patients. Frozen sections were stained by the standard indirect immunoperoxidase technique with antiserum to Ulex europaeus (E-Y Laboratories).

\section{ELECTRON MICROSCOPY}

Tissue fixed in glutaraldehyde was available from the same five patients, including material from two widely invasive tumours. These were examined after routine electron microscopy processing.

\section{Results}

Immunostaining with laminin identified acinar basement membrane and vascular endothelium. In the 11 cases also immunostained for type IV collagen an identical pattern was obtained. The endothelial component defined by laminin and type IV collagen was established by comparison with material stained with $U$ europaeus. Integrity of the

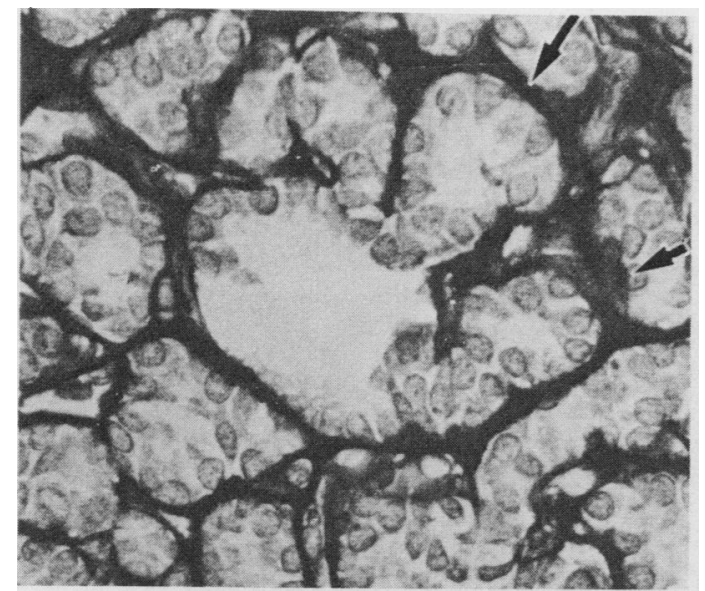

Fig. 1 Non-invasive follicular tumour showing preservation of basement membrane around follicles. Developing trabecular pattern can be seen with basement membrane distended over focal acinar cell proliferations (arrow). (Laminin.) $\times 325$. basement membrane was assessed in the range of follicular tumours. Preservation or loss could most readily be detected around the follicular structures. The follicular or trabecular architecture of the tumours was emphasised by these techniques. Endothelial staining, particularly of capsular vessels, was also examined.

\section{BASEMENT MEMBRANE (TABLE)}

Follicular tumours: non-invasive Fifteen tumours exhibited a mixed follicular and trabecular pattern, four pure follicular, and two almost entirely trabecular. In all cases basement membrane was preserved around the follicles and trabeculae. In the mixed lesions early trabecula formation could be seen. This consisted of outpouchings of basement membrane over focal proliferation of acinar cells (Fig. 1). The basement membrane in these areas was less distinct but still apparently present.

Follicular tumours: microinvasive All four cases

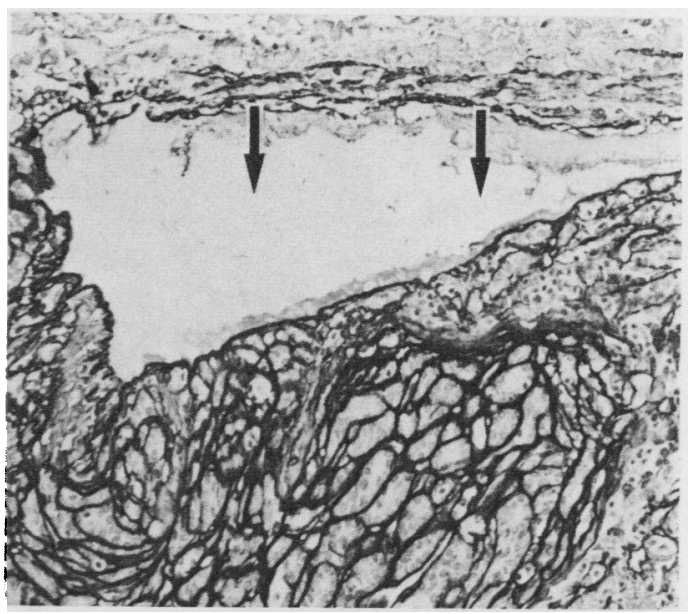

Fig. 2 Well differentiated follicular carcinoma invading a capsular vessel (arrows). Preservation of basement membrane is seen around acini in invading tumour. (Laminin.) $\times 130$. 


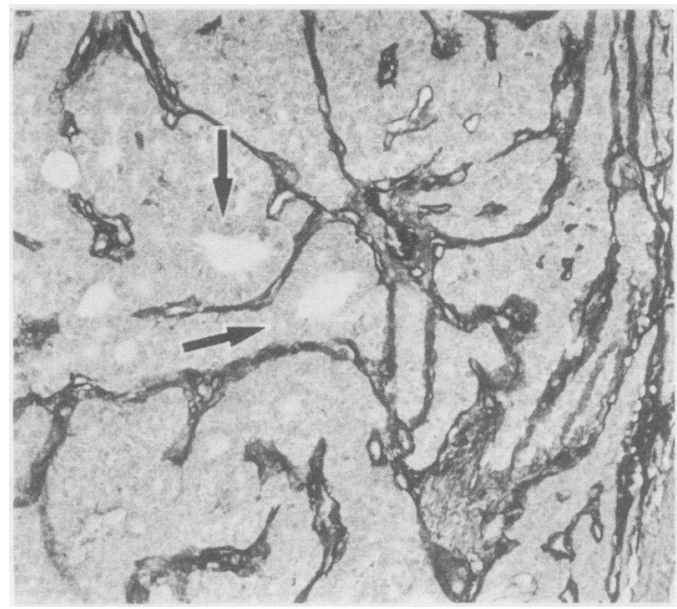

Fig. 3 Encapsulated follicular tumour showing extensive loss of basement membrane around follicular structures (arrows) and within trabeculae. Staining is confined to tumour vessels and sinusoids (Type IV collagen.) $\times 160$.

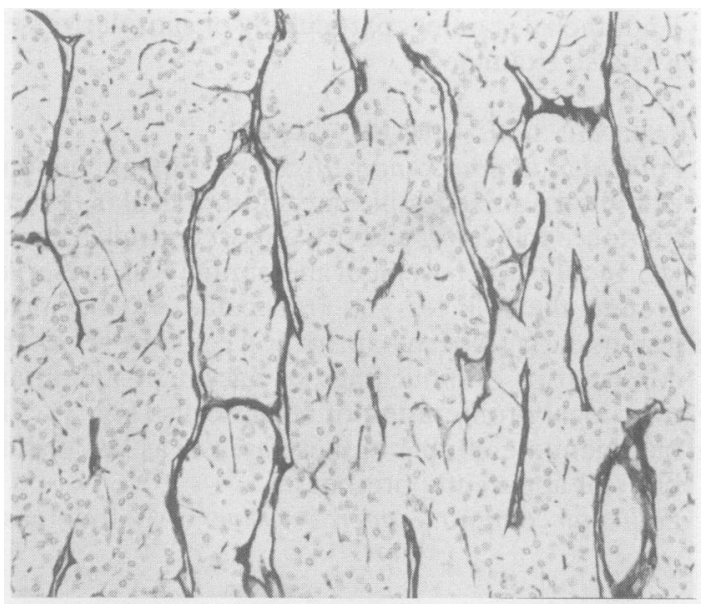

Fig. 4 Widely invasive follicular carcinoma. Tumour sinusoids are prominent. Some intervening thin strands within trabeculae may be residual acinar basement membrane. (Laminin.) $\times 160$.

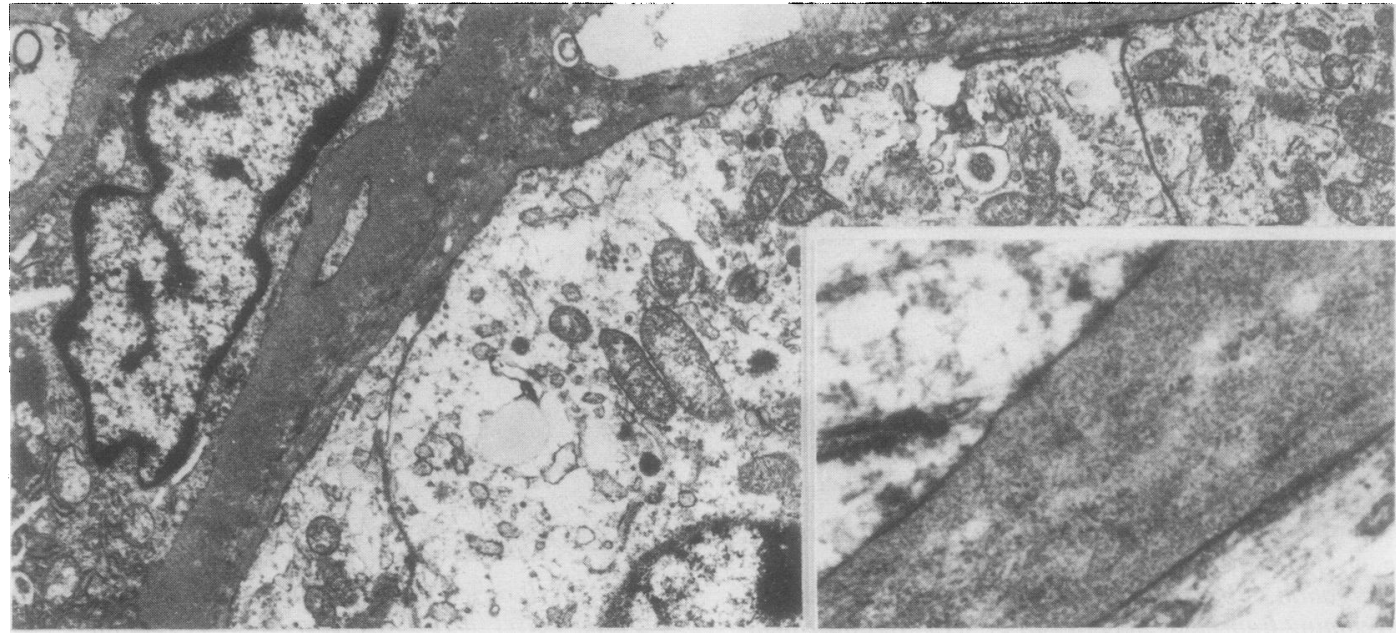

Fig. 5 Widely invasive follicular carcinoma showing loss of basal lamina around cell periphery. Inset, detail of two cell borders with intervening stroma. $\times 7000$ and 26000 . 


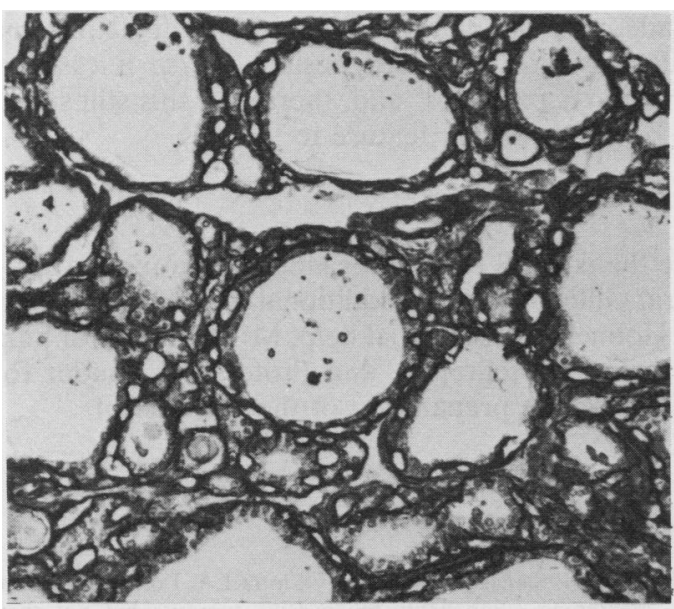

Fig. 6 Hyperplastic thyroid gland showing prominent vasculature seen as loops around periphery of follicles. (Laminin.) $\times 80$.

had a mixed trabecular and follicular pattern. Three had intact basement membrane around the trabeculae and follicles. This was seen even in areas of vascular invasion (Fig. 2). In one case, however, there was widespread loss, immunostaining of vessels providing an internal control (Fig. 3).

Follicular tumours: widely invasive Six of the nine cases had a mixed follicular and trabecular pattern, two an almost entirely trabecular arrangement, and one a solid growth pattern. Of the six cases containing recognisable follicular structures, three showed partial or complete loss around the follicles, similar to that seen in Fig. 3.

Assessing the trabecular component was more difficult. Basement membrane appeared to be intact around the periphery of most trabeculae. In many areas these were also covered by vascular sinusoids. Within the trabeculae, however, there seemed to be a considerable loss of the basement membrane, with staining confined to vessels. In some cases small amounts of residual internal basement membrane were seen as thin and incomplete strands subdividing trabeculae (Fig. 4). The case with a solid growth pattern showed only partial investment of some tumour islands.

Electron microscopy of two tumours, both of which had showed loss of laminin around the follicular structures, also showed an ultrastructural deficiency of basal lamina. Epithelial cells were seen to abut on to stroma without intervening basal lamina (Fig. 5).

\section{ENDOTHELIAL STAINING}

In normal thyroid immunostaining with laminin and type IV collagen defined both the microvasculature

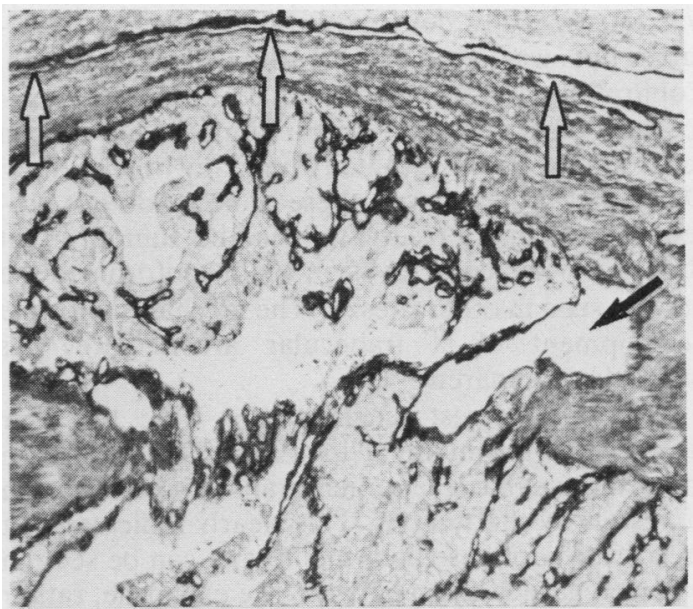

Fig. 7 Follicular tumour invading capsule. There is imminent invasion of small capsular vessel (solid arrow). Larger vessel (open arrow) is not invaded. (Laminin.) $\times 160$.

and larger vessels. Small vessels investing acini were seen as loops on the outer surface of the acinar basement membrane and were particularly prominent in the hyperplastic thyroid (Fig. 6). Small vessels were indistinct in the tumours, but larger sinusoidal vessels were apparent. Capsular vessels were readily identified, and in most cases invasion by tumour could be confirmed or excluded by capsular invasion (Fig. 7).

$U$ europaeus produced better results in frozen sections than in fixed material. In normal thyroid $U$ europaeus stained endothelial cells and emphasised the vascular network around the acini. Prominent vascularity was seen with endothelial cells again well outlined in the tumours.

\section{Discussion}

Both laminin and type IV collagen proved to be useful markers of basement membrane and endothelium in thyroid. The codistribution of laminin and type IV collagen was expected, as this has been shown in other sites. ${ }^{110}$

\section{BASEMENT MEMBRANE}

Previous studies using immunofluorescence have found some loss of basement membrane in follicular thyroid tumours, ${ }^{34}$ but it was not clear whether this could be exploited diagnostically. As minimally invasive lesions present the most difficulty the cases were subdivided by degree of invasion to correlate with the preservation or loss of basement membrane. Many of the widely invasive tumours showed a definite deficiency of basement membrane. This 
was most readily detected around follicular structures but was also seen within the trabeculae. Confirmation of this was obtained at electron microscopy in two cases. Similar findings have been recorded for invasive tumours in many other sites. ${ }^{12}$ Diagnostically, however, these tumours do not present a problem. Preservation in the tumours that show no evidence of invasion again conforms to the pattern seen in other sites. ${ }^{11}$ The mechanism for the development of a trabecular architecture was particularly apparent.

Of more interest diagnostically were the four cases with only minimal invasion. Three of these showed intact basement membrane despite invasion of vessels by the tumour. This clearly indicates that preservation of basement membrane can be seen in lesions regarded as malignant. The expansile, rather than infiltrative, nature of these early lesions is probably related to this. Electron microscopy studies have produced similar findings. ${ }^{12}$ The one case with early capsular invasion, however, in which basement membrane was clearly lost, raises interesting questions. Presumably, the prognosis would be less benign than the histology suggested. The findings, therefore, indicate that although malignancy cannot be excluded if basement membrane is intact, deficiency in an encapsulated lesion might prompt reassessment.

\section{ENDOTHELIUM}

Various methods have previously been examined for improving the detection of vascular invasion in thyroid lesions. These include stains for elastin and, more recently, factor VIII RAG. Capsular vessels, however, contain little elastic tissue,${ }^{13}$ and the value of factor VIII RAG is limited by loss of reactivity in vessels occluded by tumour. ${ }^{14}$

In our study $U$ europaeus was useful in identifying the vascular component of basement membrane defined by laminin and type IV collagen, as shown previously. ${ }^{3}$ As a marker for vascular endothelium in invasion, however, it would have little to offer, being essentially restricted to frozen sections. The value of immunostaining for components of basement membrane for detecting vascular invasion has recently been shown in breast carcinoma.? In thyroid lesions this method also seems to have some advantage over existing methods. As in the examples shown invasion of capsular vessels could be confirmed or excluded with reasonable confidence, and as this is applicable to fixed tissue, routine and retrospective cases can be examined. Much debate has centred on whether vascular invasion is essential for diagnosing malignancy in follicular lesions. Metastasis occurs rarely in cases in which only capsular invasion can be shown, ${ }^{15-17}$ but this may reflect inadequate sampling or difficulties in detecting vascular invasion. The prognosis is worse if vascular invasion is found, ${ }^{13} 18$ and, therefore, this still seems to be an important feature to identify.

We thank Mr S Forster for kindly donating the antisera, Gillian Mann for additional technical help, Mrs G Holmes for secretarial help, Ms B Jordan for help with the photography, and Professor I Lauder for help with the preparation of the manuscript.

\section{References}

' Barsky SH, Siegal GP, Janotta F, Liotta LA. Loss of basement membrane components by invasive tumours but not their benign counterparts. Lab Invest 1983;49:140-7.

${ }^{2}$ Albrechtsen R, Nielsen M, Wewer U, Engvall E, Ruoslahti E. Basement membrane changes in breast cancer detected by immunohistochemical staining for laminin. Cancer Res 1981;41:5076-81.

${ }^{3}$ Miettinen M, Virtanen I. Expression of laminin in thyroid gland tumours: an immunohistologic study. Int J Cancer 1984 34:27-30.

4 Birembaut P, Caron Y, Adnet J-J, Foidart JM. Usefulness of basement membrane markers in tumoural pathology.J Pathol 1985; 145: 283-96.

${ }^{5}$ Foidart JM, Bere EW, Yaar M, et al. Distribution andO immunoelectron microscopic localisation of laminin, a non coll lagenous basement membrane component. Lab Invest 1980 42: 336-42.

- Yaoita H, Foidart JM, Katz SI. Localization of the collagenous' component in skin basement membrane. $J$ Invest Dermatol 1978; 70:191-3.

' Bettelheim R, Mitchell D, Gusterson BA. Immunocytochemistry in the identification of vascular invasion in breast cancer.J Clin Pathol 1984;37:364-6.

${ }^{8}$ Ekblom P, Miettinen M, Rapola J, Foidart JM. Demonstration of laminin, a basement membrane glycoprotein in routinely processed formalin-fixed human tissues. Histochemistry 1982; 75:301-7.

' Forster SJ, Talbot IC, Critchley DR. Laminin and fibronectin in rectal adenocarcinoma: relationship to tumour grade, stage and metastasis. $\mathrm{Br} J$ Cancer 1984;50:51-61.

${ }^{10}$ Burtin P, Chavanel G, Foidart JM, Martin E. Antigens of the basement membrane and the peritumoral stroma in human colonic adenocarcinomas: an immunofluorescence study. Int $J$ Cancer 1982;30:13-20.

" Ekblom P, Miettinen M, Forsman L, Andersson LC. Basement membrane and apocrine epithelial antigens in differential diagnosis between tubular carcinoma and sclerosing adenosis of the breast. J Clin Pathol 1984;37:357-63.

12 Johanessen JV, Gould VE, Jao W. The fine structure of human thyroid cancer. Hum Pathol 1978;9:385-400.

${ }^{13}$ Hazard JB, Kenyon R. Encapsulated angioinvasive carcinoma (angioinvasive adenoma) of thyroid gland. Am J Clin Pathol 1954;24:755-66.

${ }_{14}$ Harach HR, Jasani B, Williams ED. Factor VIII as a marker of endothelial cells in follicular carcinoma of the thyroid. $J$ Clin Pathol 1983;36: 1050-4.

is Kahn NF, Perzin KH. Follicular carcinoma of the thyroid: an evaluation of the histologic criteria used for diagnosis. Pathol Ann 1983; Part 1: 221-53.

16 Schroder S, Pfannschmidt N, Dralle H, Arps H, Bocker W. The 
encapsulated follicular carcinoma of thyroid. A clinicopathologic study of 35 cases. Virchows Arch (Pathol Anat) $1984 ; 402: 259-73$.

${ }^{17}$ Evans HL. Follicular neoplasms of the thyroid. A study of 44 cases followed for a minimum of 10 years, with emphasis on differential diagnosis. Cancer 1984;54:535-40.

${ }^{18}$ Cady B, Sedgwick CE, Meissner WA, Bookwalter JR, Romagosa V, Werber J. Changing clinical pathologic therapeutic and sur- vival patterns in differentiated thyroid carcinoma. Ann Surg 1976; 184: 541-53.

Requests for reprints to: $\mathrm{Dr} \mathrm{CH}$ Kendall, Department of Pathology, Clinical Sciences Building, Leicester Royal Infirmary, PO Box 65, Leicester LE2 7LX, England. 